The generating hypothesis in the derived category of a ring

Hovey, Mark and Lockridge, Keir and Puninski, Gena 2007

MIMS EPrint: 2007.180

Manchester Institute for Mathematical Sciences

School of Mathematics

The University of Manchester

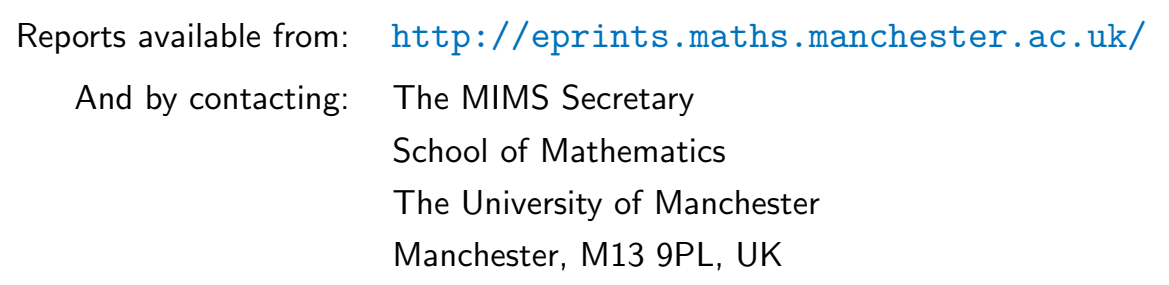

ISSN 1749-9097 


\title{
The generating hypothesis in the derived category of a ring
}

\author{
Mark Hovey · Keir Lockridge • Gena Puninski
}

Received: 4 October 2006 / Accepted: 27 November 2006 /

Published online: 8 February 2007

(C) Springer-Verlag 2007

\begin{abstract}
We show that a strong form (the fully faithful version) of the generating hypothesis, introduced by Freyd in algebraic topology, holds in the derived category of a ring $R$ if and only if $R$ is von Neumann regular. This extends results of the second author (J. Pure Appl. Algebra 208(2), 2007). We also characterize rings for which the original form (the faithful version) of the generating hypothesis holds in the derived category of $R$. These must be close to von Neumann regular in a precise sense, and, given any of a number of finiteness hypotheses, must be von Neumann regular. However, we construct an example of such a ring that is not von Neumann regular and therefore does not satisfy the strong form of the generating hypothesis.
\end{abstract}

\section{Introduction}

The generating hypothesis was introduced by Peter Freyd [4] in algebraic topology, where it is the assertion that any map $f: X \rightarrow Y$ of finite spectra that is 0 on stable homotopy groups is in fact null homotopic. The generating hypothesis is widely considered to be one of the most important and difficult problems in stable homotopy theory. It has many implications for the structure of the stable homotopy ring $\pi_{*} S^{0}$ of the sphere, implying for example that it is totally non-coherent [4] and that the

M. Hovey $(\varangle) \cdot$ K. Lockridge

Department of Mathematics, Wesleyan University, Middletown, CT 06459, USA

e-mail: hovey@member.ams.org

K. Lockridge

e-mail: keir@alumni.rice.edu

G. Puninski

Department of Mathematics, University of Manchester, Booth Street East,

Manchester M13 PL, UK

e-mail: gpuninski@maths.man.ac.uk 
$p$-completion $\pi_{*} S_{p}^{0}$ is a self-injective ring [7]. Somewhat surprisingly, Freyd proved that the generating hypothesis in fact implies that the map

$$
[X, Y] \rightarrow \operatorname{Hom}_{\pi_{*} S^{0}}\left(\pi_{*} X, \pi_{*} Y\right)
$$

from maps of finite spectra to maps of their stable homotopy modules is not only injective but also surjective. That is, the generating hypothesis implies that the stable homotopy functor is fully faithful on finite spectra.

One approach to understanding the generating hypothesis is to look at analogous questions in other categories. Following the second author [9], we say that a ring $R$ satisfies the generating hypothesis if whenever $f: X \rightarrow Y$ is a map of perfect complexes in the derived category $\mathcal{D}(R)$ of $R$ and $H_{*} f=0$, then $f=0$. Recall that a perfect complex is a bounded chain complex of finitely generated projective (right) modules, and that $f=0$ in $\mathcal{D}(R)$ exactly when $f$ is chain homotopic to 0 (for maps of perfect complexes). Perfect complexes are the algebraic analogue of finite spectra, as they are the small objects in $\mathcal{D}(R)$. Thus, $R$ satisfies the generating hypothesis exactly when the homology functor is faithful on perfect complexes. Let us also say that $R$ satisfies the strong generating hypothesis if the homology functor is fully faithful on perfect complexes.

The second author noticed [9, Sect. 4] that the homology functor is faithful on all of $\mathcal{D}(R)$ if and only if all right $R$-modules are projective; that is, if and only if $R$ is semisimple. Since perfect complexes are the small objects of $\mathcal{D}(R)$ and finitely presented modules are the small $R$-modules, it is natural to conjecture (as the second author did in [9]) that the homology functor is faithful on perfect complexes (that is, $R$ satisfies the generating hypothesis) if and only if all finitely presented right $R$-modules are projective; that is, if and only if $R$ is von Neumann regular. The second author verified that all von Neumann regular rings do satisfy the generating hypothesis, and proved that if $R$ satisfies the generating hypothesis and is either commutative or right coherent, then $R$ is von Neumann regular [9].

In this paper, we first prove that $R$ satisfies the strong generating hypothesis if and only if $R$ is von Neumann regular. We then consider the generating hypothesis, in effect asking whether the generating hypothesis implies the strong generating hypothesis. We prove that $R$ satisfies the generating hypothesis if and only if all short exact sequences of finitely presented modules split, and all submodules of flat modules are flat. This makes $R$ close to von Neumann regular, and in fact if $R$ is local or satisfies one of several finiteness hypotheses it forces $R$ to be von Neumann regular. However, we construct an example of a ring that satisfies the generating hypothesis but is not von Neumann regular. Over this ring, then, the homology functor is faithful on perfect complexes but not full.

The authors would like to thank Grigory Garkusha for many helpful discussions, and the referee for simplifying some of our arguments.

All $R$-modules $M$ will be right $R$-modules in this paper, so that, for example, $\mathcal{D}(R)$ is the unbounded derived category of right $R$-modules. The differential $d$ in a chain complex $P$ will lower dimension, so that $d_{n}: P_{n} \rightarrow P_{n-1}$. We will denote ker $d_{n}$ by $Z_{n} P$ and im $d_{n}$ by $B_{n-1} P$. If $M$ is an $R$-module, then $D^{n}(M)$ denotes the complex which is $M$ in degree $n$ and $n-1$ and 0 elsewhere, with $d_{n}$ being the identity. $S^{n} M$ denotes the complex that is $M$ in degree $n$ and 0 elsewhere. 


\section{The strong generating hypothesis}

We begin by recalling the second author's characterization of semisimple rings.

Lemma 1.1 Let $P$ be an object of $\mathcal{D}(R)$. Assume $H_{n} P$ is projective for all $n$. Then $P \cong \bigoplus_{n} S^{n}\left(H_{n} P\right)$. Furthermore, for every object $Q \in \mathcal{D}(R)$, the natural map

$$
[P, Q] \rightarrow \operatorname{Hom}_{R}\left(H_{*} P, H_{*} Q\right)
$$

is an isomorphism.

Proof If $M$ is a projective $R$-module and $Q$ is a complex, chain homotopy classes of chain maps from $S^{n} M$ to $Q$ are isomorphic to $\operatorname{Hom}_{R}\left(M, Z_{n} Q\right) / \operatorname{Hom}_{R}\left(M, B_{n} Q\right)$. This embeds in $\operatorname{Hom}_{R}\left(M, H_{n} Q\right)$, and the cokernel is a subgroup of $\operatorname{Ext}_{R}^{1}\left(M, B_{n} Q\right)=0$. Hence, if $M$ is projective, then

$$
\left[S^{n} M, Q\right] \cong \operatorname{Hom}_{R}\left(M, H_{n} Q\right) .
$$

In particular, the identity map of $H_{n} P$ lifts to a map $S^{n}\left(H_{n} P\right) \rightarrow P$. This gives us a map

$$
\bigoplus_{n} S^{n}\left(H_{n} P\right) \rightarrow P
$$

that is a homology isomorphism, hence an isomorphism in $\mathcal{D}(R)$. The second part now follows easily.

Proposition 1.2 $A$ ring $R$ is semisimple if and only if the homology functor is faithful on $\mathcal{D}(R)$. Furthermore, in this case, the homology functor is in fact fully faithful on $\mathcal{D}(R)$.

Proof If $R$ is semisimple, then Lemma 1.1 implies that homology is fully faithful. For the converse, take two $R$-modules $M$ and $N$, and a projective resolution $P$ of $M$. Then an element of $\operatorname{Ext}^{S}(M, N)$ is represented by a map from $P$ to $N$, thought as a complex concentrated in degree $s$. This map is necessarily 0 in homology when $s>0$. Thus $\operatorname{Ext}^{S}(M, N)=0$ for all $s>0$ and all $M, N$, so every $R$-module is projective and $R$ is semisimple.

The analogue for the generating hypothesis is the following theorem.

Theorem 1.3 $A$ ring $R$ satisfies the strong generating hypothesis if and only if $R$ is von Neumann regular. In this case, the natural map

$$
[P, Q] \rightarrow \operatorname{Hom}_{R}\left(H_{*} P, H_{*} Q\right)
$$

is an isomorphism for all perfect complexes $P$ and arbitrary complexes $Q$.

Recall that $R$ is von Neumann regular if and only if, for every $x \in R$, there is a $y \in R$ with $x=x y x$. The standard reference for von Neumann regular rings is [6]; the book [8] takes an approach based on module categories, so contains some different and useful results about von Neumann regular rings. A standard characterization is that $R$ is von Neumann regular if and only if all $R$-modules are flat if and only if all finitely presented $R$-modules are projective. Note as well that von Neumann regular rings are coherent. 
Proof Suppose $R$ satisfies the strong generating hypothesis. Then,

$$
\operatorname{ann}_{\ell} \operatorname{ann}_{r}(R x)=R x
$$

for all $x \in R$, by [9, Proposition 2.7]. Now take $x \in R$, and consider the perfect complex $P$ with $P_{i}=R$ if $i=0,1$ and $P_{i}=0$ otherwise, with the differential $P_{1} \rightarrow P_{0}$ being left multiplication by $x$. This complex has $H_{0}(P)=R / x R$ and $H_{1}(P)=\operatorname{ann}_{r}(x)$. By the strong generating hypothesis, there exists a chain map $\phi: P \rightarrow P$ such that $H_{1}(\phi)=0$ and $H_{0}(\phi)=1$, the identity of $R / x R$. Translating, this means there exist elements $a, b \in R$ such that $x a=b x$ with $a \in \operatorname{ann}_{\ell} \operatorname{ann}_{r}(x)$ (so that $\left.H_{1}(\phi)=0\right)$ and $b=1+x c$ for some $c \in R$ (so that $H_{0}(\phi)=1$ ). But then $a=d x$ for some $d \in R$, so we have

$$
x d x=x a=b x=(1+x c) x=x+x c x .
$$

This means that $x=x(d-c) x$. Since $x$ was arbitrary, $R$ is von Neumann regular.

Conversely, suppose $R$ is von Neumann regular, and $P$ is a perfect complex. Since $R$ is coherent, $H_{n} P$ is finitely presented for all $n$, and thus must be projective. Lemma 1.1 then completes the proof.

Recall from [9] that if $R$ is either commutative or right coherent and $R$ satisifes the generating hypothesis, then $R$ is von Neumann regular. Hence we get the following corollary.

Corollary 1.4 If $R$ is either commutative or right coherent, then $R$ satisfies the generating hypothesis if and only if $R$ satisfies the strong generating hypothesis.

Recall that a full subcategory of a triangulated category is called thick if it is closed under shifts, retracts, and cofibers; the thick subcategory generated by $R$ consists of the perfect complexes.

The special case of the following result when $R$ is either commutative or right coherent is proved in [9].

Corollary 1.5 A ring $R$ satisfies the strong generating hypothesis if and only if, in $\mathcal{D}(R)$, the thick subcategory generated by $R$ is the collection of retracts of finite coproducts of suspensions of $R$.

This corollary indicates how different the stable homotopy category must be from the derived category of a ring if Freyd's generating hypothesis is to be true, since there are many finite spectra that are not retracts of finite coproducts of suspensions of the sphere.

\section{Rings that satisfy the generating hypothesis}

Having dealt with the strong generating hypothesis, we now turn our attention to the generating hypothesis. The objective of this section is to prove the following theorem.

Theorem 2.1 $A$ ring $R$ satisfies the generating hypothesis if and only if $R$ has weak dimension at most 1 and all finitely presented $R$-modules are FP-injective.

Weak dimension at most 1 is of course equivalent to the statement that submodules of flat modules are flat. Recall that a module $M$ is said to be FP-injective if

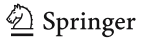


$\operatorname{Ext}^{1}(F, M)=0$ for all finitely presented modules $F$; thus all finitely presented modules are FP-injective if and only if all short exact sequences of finitely presented modules split. FP-injective modules seem to have been introduced in [14]; a good guide to the literature can be found in [3, Chap. 6]. An FP-injective module is sometimes called absolutely pure, because $M$ is FP-injective if and only if every short exact sequence

$$
0 \rightarrow M \rightarrow N \rightarrow P \rightarrow 0
$$

is pure (that is, remains exact upon tensoring with any left $R$-module). See [8, Theorem 4.89(5)] for a proof of this equivalence.

To compare the rings of Theorem 2.1 with von Neumann regular rings, the following lemma is helpful.

Lemma 2.2 $A$ ring $R$ is von Neumann regular if and only if every $R$-module is $F P$ injective.

This lemma is well-known, but does not appear in [6] or [8], so we include the proof for the convenience of the reader.

Proof Suppose $R$ is von Neumann regular, and $M$ is an $R$-module. Choose a short exact sequence $\mathcal{E}$

$$
0 \rightarrow M \rightarrow I \rightarrow N \rightarrow 0
$$

where $I$ is injective. Since $N$ is necesarily flat, this sequence is pure [8, Theorem 4.85]. Hence, if $F$ is finitely presented, $\operatorname{Hom}(F, \mathcal{E})$ is still exact [8, Theorem 4.89(5)], and so $\operatorname{Ext}^{1}(F, M)=0$ and $M$ is FP-injective.

Conversely, if every module is FP-injective, another application of [8, Theorem 4.89 (5)] shows that every short exact sequence is pure. Then [8, Theorem 4.85] shows that every module is flat, as required.

We now begin the proof of Theorem 2.1. Our first task is to characterize the homology groups of perfect complexes.

Proposition 2.3 Suppose $R$ is a ring. An $R$-module $M$ is a homology module of a perfect complex of $R$-modules if and only if there exists a finitely presented module $F$ such that $M$ embeds in $F$ and the quotient $F / M$ embeds in a projective module. Furthermore, in this case, there is a perfect complex $P$ such that $P_{n}=0$ unless $n=0,1,2$ and $M=H_{1} P$.

Proof Suppose $M=H_{n} P$, where each $P_{i}$ is a finitely generated projective module. Then we have a short exact sequence

$$
0 \rightarrow M \rightarrow P_{n} / B_{n} P \stackrel{d_{n}}{\rightarrow} B_{n-1} P \rightarrow 0
$$

$P_{n} / B_{n} P$ is finitely presented and $B_{n-1} P$ embeds in the projective module $P_{n-1}$.

Conversely, suppose $M$ embeds in the finitely presented module $F$ and the quotient $F / M$ embeds in the projective module $P_{0}$, which we can assume is finitely generated since $F$ is so. Choose a presentation

$$
P_{2} \stackrel{d_{2}}{\rightarrow} P_{1} \stackrel{p}{\rightarrow} F \rightarrow 0
$$


of $F$, where $P_{1}$ and $P_{2}$ are finitely generated projectives. Define the map $d_{1}: P_{1} \rightarrow P_{0}$ to be the composite

$$
P_{1} \stackrel{p}{\rightarrow} F \rightarrow F / M \rightarrow P_{0} .
$$

This defines a three-term perfect chain complex $P$. Pulling back the presentation of $F$ through the inclusion $M \rightarrow F$ shows that $H_{1} P \cong M$.

We now take a significant step towards Theorem 2.1 by showing how FP-injective modules arise.

Theorem 2.4 Let $R$ be a ring, and let $Q$ be an arbitrary object of $\mathcal{D}(R)$. Then the generating hypothesis with target $Q$ is true in $\mathcal{D}(R)$ if and only if $H_{n} Q$ is FP-injective for all $n$. In particular, $R$ satisfies the generating hypothesis if and only if all homology modules of perfect complexes are FP-injective.

The generating hypothesis with target $Q$ is the statement that any map $f: P \rightarrow Q$ in $\mathcal{D}(R)$ where $P$ is a perfect complex and $H_{*} f=0$ has $f=0$. So $R$ satisfies the generating hypothesis if and only if $R$ satisfies the generating hypothesis with target $Q$ for all perfect complexes $Q$.

Note in particular that this theorem and Lemma 2.2 imply that $R$ satisfies the generating hypothesis with target $Q$ for all (not necessarily perfect) $Q$ if and only if $R$ is von Neumann regular.

Proof Suppose first that the generating hypothesis with target $Q$ holds, and consider a finitely presented module $F$ and an integer $n$. Choose a finite presentation

$$
P_{n} \stackrel{d_{n}}{\rightarrow} P_{n-1} \rightarrow F \rightarrow 0
$$

of $F$, so that, by letting $P_{i}=0$ for $i \neq n, n-1$, we get a perfect complex $P$ with $H_{n-1} P=F$. To prove that

$$
\operatorname{Ext}^{1}\left(F, H_{n} Q\right)=0,
$$

it suffices to show that any map

$$
f: P_{n} / Z_{n} P \rightarrow H_{n} Q
$$

extends to a map $g: P_{n-1} \rightarrow H_{n} Q$ with $g \overline{d_{n}}=f$, where $\overline{d_{n}}$ is the map induced by $d_{n}$.

Since $P_{n}$ is projective, there is a map $\phi_{n}: P_{n} \rightarrow Q_{n}$ such that the composite

$$
P_{n} \stackrel{\phi_{n}}{\rightarrow} Q_{n} \stackrel{q}{\rightarrow} Q_{n} / B_{n} Q
$$

is the composite

$$
P_{n} \stackrel{p}{\rightarrow} P_{n} / Z_{n} P \stackrel{f}{\rightarrow} H_{n} Q \stackrel{i}{\rightarrow} Q_{n} / B_{n} Q .
$$

Now let $\phi_{n-1}: P_{n-1} \rightarrow Q_{n-1}$ be the zero map. Then $\phi: P \rightarrow Q$ is a chain map. Indeed, write $d_{n}: Q_{n} \rightarrow Q_{n-1}$ as $d_{n}=r q$, where $r: Q_{n} / B_{n} Q \rightarrow Q_{n-1}$. Then

$$
d_{n} \phi_{n}=r q \phi_{n}=r i f p=0
$$

since $r i=0$. Furthermore, $\phi$ induces the zero map on homology, because if $x \in Z_{n} P$, then $q \phi_{n} x=0$, so $\phi_{n} x$ is a boundary. 
If the generating hypothesis is true, then $\phi$ must be chain homotopic to 0 . This gives us maps $D_{n-1}: P_{n-1} \rightarrow Q_{n}$ and $D_{n}: P_{n} \rightarrow Q_{n+1}$ such that $d_{n} D_{n-1}=0$ and $D_{n-1} d_{n}+d_{n+1} D_{n}=\phi_{n}$. Since $d_{n}: Q_{n} \rightarrow Q_{n-1}$ factors through $Q_{n} / B_{n} Q$ as $d_{n}=\overline{d_{n}} q$, we conclude that $\overline{d_{n}} q D_{n-1}=0$, so there exists a map $g: P_{n-1} \rightarrow H_{n} Q$ such that $i g=q D_{n-1}$. Of course, we claim that $g$ is the desired extension. To see this, apply $q$ to the relation

$$
D_{n-1} d_{n}+d_{n+1} D_{n}=\phi_{n}
$$

to get

$$
q D_{n-1} d_{n}=\text { ifp or } i g d_{n}=i f p .
$$

Writing $d_{n}=\overline{d_{n}} p$ and using the fact that $i$ is a monomorphism and $p$ is an epimorphism, we conclude that $g \overline{d_{n}}=f$, as required.

Now suppose that every homology group of $Q$ is FP-injective, and $\phi: P \rightarrow Q$ is a map of chain complexes that induces 0 on homology. We will construct a chain homotopy $D_{n}: P_{n} \rightarrow Q_{n+1}$ such that $d_{n+1} D_{n}+D_{n-1} d_{n}=\phi_{n}$ by induction on $n$. Our induction hypothesis will be that we have constructed $D_{i}$ for $i \leq n-1$ and that $\phi_{n}-D_{n-1} d_{n}$, which is a map from $P_{n}$ to $Q_{n}$, in fact lands in the boundaries $B_{n} Q$. Getting started is easy since $P$ is bounded below. For the induction step, our hypothesis gives us the commutative square below,

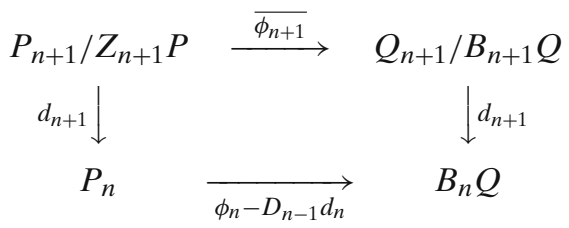

where $\overline{\phi_{n+1}}$ exists because $\phi$ is zero on homology, so must take cycles to boundaries. We will construct a lifting $\overline{D_{n}}: P_{n} \rightarrow Q_{n+1} / B_{n+1} Q$ in this square. First of all, there is obviously a map

$$
E_{n}: P_{n} \rightarrow Q_{n+1} / B_{n+1} Q
$$

such that $d_{n+1} E_{n}=\phi_{n}-D_{n-1} d_{n}$, simply because $P_{n}$ is projective. Then

$$
d_{n+1}\left(\overline{\phi_{n+1}}-E_{n} d_{n+1}\right)=d_{n+1} \overline{\phi_{n+1}}-\phi_{n} d_{n+1}+D_{n-1} d_{n} d_{n+1}=0 .
$$

Hence $\overline{\phi_{n+1}}-E_{n} d_{n+1}$ is a map from $P_{n+1} / Z_{n+1} P$ to $H_{n+1} Q$. Since $H_{n+1} Q$ is FP-injective, there is a map $F_{n}: P_{n} \rightarrow H_{n+1} Q$ such that $F_{n} d_{n+1}=\overline{\phi_{n+1}}-E_{n} d_{n+1}$. Hence

$$
\overline{D_{n}}=E_{n}+F_{n}: P_{n} \rightarrow Q_{n+1} / B_{n+1} Q
$$

defines a lift in our commutative square.

We now choose $D_{n}: P_{n} \rightarrow Q_{n+1}$ lifting $\overline{D_{n}}$, which we can do because $P_{n}$ is projective. Then one can easily check that $d_{n+1} D_{n}=\phi_{n}-D_{n-1} d_{n}$, and also, because $\overline{D_{n}} d_{n+1}=\overline{\phi_{n+1}}$, that $\phi_{n+1}-D_{n} d_{n+1}$ lands in $B_{n+1} Q$. This completes the induction step and the proof.

We can now prove Theorem 2.1.

Proof of Theorem 2.1 Suppose the generating hypothesis holds in $\mathcal{D}(R)$. In view of Theorem 2.4, we need only show that $R$ has weak dimension at most 1 . Since 
Tor $_{*}(-, M)$ commutes with direct limits, it suffices to show that the flat dimension of any finitely presented module is at most 1 . Since any finitely presented module is a homology group of a perfect complex, it is enough to show that the cycles $Z_{n} P$ and the boundaries $B_{n} P$ are flat for all perfect complexes $P$ and integers $n$. But $Z_{n} P$ is itself a homology group of a perfect complex (simply truncate $P$ by replacing all the $P_{i}$ for $i>n$ by 0 ), and so Theorem 2.4 implies that $Z_{n} P$ is FP-injective. This means that the short exact sequence

$$
0 \rightarrow Z_{n} P \rightarrow P_{n} \rightarrow B_{n-1} P \rightarrow 0
$$

is pure. Now choose a left $R$-module $M$ and apply $-\otimes_{R} M$ to this short exact sequence. By purity, it remains exact, and so the Tor long exact sequence shows that $\operatorname{Tor}_{1}^{R}\left(B_{n-1} P, M\right)=0$. Since $M$ was arbitrary, $B_{n-1} P$ is flat. But then $Z_{n} P$, as a kernel of a surjection of flat modules, is also flat.

Conversely, assume $R$ has weak dimension at most 1 and all finitely presented $R$-modules are FP-injective. We need to show that an arbitrary homology group $M$ of a perfect complex is FP-injective, by Theorem 2.4. By Proposition 2.3, there is a finitely presented module $F$ and an exact sequence

$$
0 \rightarrow M \rightarrow F \rightarrow F / M \rightarrow 0,
$$

where $F / M$ embeds in a projective module. Since $R$ has weak dimension at most 1 , $F / M$ is flat. But then the above exact sequence is pure [8, Theorem 4.85]. Applying $\operatorname{Hom}_{R}(N,-)$ to this sequence we get a long exact sequence

$$
\begin{aligned}
0 \rightarrow \operatorname{Hom}_{R}(N, M) & \rightarrow \operatorname{Hom}_{R}(N, F) \rightarrow \operatorname{Hom}_{R}(N, F / M) \\
& \rightarrow \operatorname{Ext}_{R}^{1}(N, M) \rightarrow \operatorname{Ext}_{R}^{1}(N, F) \rightarrow \cdots
\end{aligned}
$$

If $N$ is finitely presented, though, the map $\operatorname{Hom}_{R}(N, F) \rightarrow \operatorname{Hom}_{R}(N, F / M)$ is surjective, since our original sequence is pure [8, Theorem 4.89(5)]. By hypothesis, $\operatorname{Ext}_{R}^{1}(N, F)=0$, so we conclude that $\operatorname{Ext}_{R}^{1}(N, M)=0$. Thus $M$ is FP-injective.

\section{Examples and counterexamples}

In this section, we give conditions under which rings that satisfy the generating hypothesis must be von Neumann regular, and also give an example of a ring that satisfies the generating hypothesis yet is not von Neumann regular, and thus does not satisfy the strong generating hypothesis.

Theorem 3.1 A ring $R$ is von Neumann regular if and only if the generating hypothesis holds in $\mathcal{D}(R)$ and finitely generated flat submodules of projective right $R$-modules are projective.

Proof Assume that the generating hypothesis holds in $\mathcal{D}(R)$ and finitely generated flat submodules of projectives are projective. Suppose $M$ is finitely presented, so that we have a short exact sequence

$$
0 \rightarrow K \rightarrow P \rightarrow M \rightarrow 0
$$

where $P$ is a finitely generated projective and $K$ is finitely generated. Since $R$ has weak dimension $\leq 1$ by Theorem $2.1, K$ is a finitely generated flat submodule of a projective module. By hypothesis, then, $K$ is projective, and hence finitely presented. Since $M$ 
is FP-injective by Theorem 2.1, our short exact sequence splits, and $M$ is projective. Thus every finitely presented module is projective, so $R$ is von Neumann regular.

Conversely, if $R$ is von Neumann regular, then any finitely generated submodule of a projective module is projective [8, Example 2.32(d)].

This immediately gives the following corollary, implicit in [9].

Corollary 3.2 A ring $R$ is von Neumann regular if and only if $R$ satisfies the generating hypothesis and is right coherent.

Proof If $R$ is right coherent, then a finitely generated submodule of a projective module is finitely presented. If it is also flat, then it is projective.

There are a great many rings where finitely generated flat modules are known to be projective [11]. The following theorem contains some cases of this, which are somewhat less satisfactory since not all von Neumann regular rings satisfy the hypotheses.

Theorem 3.3 Suppose the generating hypothesis holds in $\mathcal{D}(R)$ and one of the following hypotheses holds.

(1) $R$ is local (unique maximal right ideal).

(2) $R$ is semiperfect (every finitely generated module has a projective cover).

(3) $R$ is reduced (no nonzero nilpotents) and has finite uniform dimension ( $R$ contains no infinite direct sum of nonzero right ideals).

(4) $R$ has zero Jacobson radical and finite uniform dimension.

(5) $R$ is right nonsingular (the only element whose right annihilator is essential in $R$ is 0$)$ and has finite uniform dimension.

(6) $R$ is simple (no nontrivial two-sided ideals) and has finite uniform dimension.

Then $R$ is von Neumann regular.

Note that these conditions may not all be independent of each other. For example, the authors suspect that if $R$ is both right FP-injective (as it must be if it satisfies the generating hypothesis) and has finite uniform dimension, then $R$ may have to be semiperfect.

Proof For a local, semiperfect, or right nonsingular ring with finite uniform dimension, every finitely generated flat module is projective; the local case is due to Endo and can be found in [8, Theorem 4.38]. The semiperfect case is due to Bass and is [8, Exercise 4.21]. The right nonsingular case is due to Sandomierski [13, Corollary 1, p. 228]. Every reduced ring is right nonsingular by [8, Lemma 7.8]; since the singular elements form a two-sided ideal, every simple ring is also right nonsingular [8, Section 7A]. If $R$ is FP-injective, or in fact only has $\operatorname{Ext}^{1}(R / a R, R)=0$ for all $a \in R$, then having zero Jacobson radical is equivalent to being right nonsingular, by $[10$, Theorem 2.1].

Not every von Neumann regular ring has finite uniform dimension. They all, however, are right nonsingular [8, Corollary 7.7]. This leads to the following theorem.

Theorem 3.4 $A$ ring $R$ is von Neumann regular if and only if it satisfies the generating hypothesis, is right nonsingular, and its maximal right ring of quotients $Q$ is a flat left $R$-module. 
The maximal right ring of quotients of $R$ is the endomorphism ring of the injective hull of $R$ as a right $R$-module, and is much studied in ring theory. See [8, Sect. 13] for an introduction. When $R$ is right nonsingular, $Q$ is just equal to the injective hull of $R$.

Proof Sandomierski [13, Theorem 2.9] proves that if $R$ is right nonsingular and the maximal right ring of quotients $Q$ is flat as a left $R$-module, then finitely generated flat submodules of free $R$-modules (and hence also of projective $R$-modules) are projective. Theorem 3.1 completes the proof.

Theorem 3.5 There exists a ring $S$ that satisfies the generating hypothesis but is not von Neumann regular.

Of course, such a ring will not satisfy the strong generating hypothesis. Before proving this theorem, we need the following lemmas.

Lemma 3.6 Every principal right ideal of a ring $R$ is flat if and only if whenever $a b=0$ in $R$ there is an $x \in R$ such that $a x=0$ and $x b=b$.

Proof Consider the short exact sequence

$$
0 \rightarrow \operatorname{ann}_{r} a \rightarrow R \stackrel{a \times}{\longrightarrow} a R \rightarrow 0 .
$$

By [8, Theorem 4.23], $a R$ is flat if and only if for every $b \in \operatorname{ann}_{r} a$, there is a map $\theta: R \rightarrow \operatorname{ann}_{r}(a)$ with $\theta(b)=b$. Translating, this means that $a R$ is flat if and only if whenever $a b=0$, there is an $x$ such that $a x=0$ and $x b=b$.

Lemma 3.7 $A$ ring $R$ has weak dimension $\leq 1$ if and only if for every integer $m$ and every pair of $m \times m$ matrices $A, B$ over $R$ with $A B=0$, there is an $m \times m$ matrix $X$ over $R$ such that $A X=0$ and $X B=B$.

Proof In view of Lemma 3.6, the matrix condition of this lemma is equivalent to every principal right ideal of $M_{m}(R)$ being flat, for all $m \geq 1$. We will use the Morita equivalence between $R$ and $M_{m}(R)$ to prove that this is equivalent to $R$ having weak dimension $\leq 1$. Indeed, if $R$ has weak dimension $\leq 1$, so does $M_{m}(R)$ [8, p. 481], and so every ideal of $M_{m}(R)$ is flat.

Conversely, suppose every principal right ideal of $M_{m}(R)$ is flat for all $m \geq 1$. Suppose $I$ is an $m$-generated right ideal of $R$. Then $I$ corresponds under the Morita equivalence to a principal right ideal of $M_{m}(R)$ [8, Remark 17.23(C)]. This principal ideal is flat, and so $I$ is flat as well, since Morita equivalences preserve flatness [8, p. 481].

Hence all finitely generated ideals of $R$ are flat. Since Tor commutes with direct limits, all ideals of $R$ are flat. But then $R$ has weak dimension $\leq 1$ [8, Lemma 4.66].

Proof of Theorem 3.5 We will use the method of [12], who introduce and study indiscrete rings. For us, the salient property of indiscrete rings is that all finitely presented modules over an indiscrete ring are FP-injective [12, Theorem 2.4]. Thus, we must find an indiscrete ring that also has weak dimension one. The construction given in [12, p. 359] begins with a finite-dimensional algebra $R$ of finite representation type over an infinite field $F$. Because we want to end up with something of weak dimension one, we will take $R$ to have right (and left) global dimension 1. For example, we can 
take $R$ to be the ring of $2 \times 2$ upper triangular matrices over $F$, which is a classical example of a ring of right (and left) global dimension 1 that is not von Neumann regular [8, Example 2.36].

The method of [12] is then to construct a map $\tau: R \rightarrow M_{n} R$ and then let $S=R_{\tau}$ be the direct limit

$$
S=R_{\tau}=\operatorname{colim}\left(R \stackrel{\tau}{\rightarrow} M_{n} R \stackrel{M_{n} \tau}{\longrightarrow} M_{n^{2}} R \stackrel{M_{n^{2}} \tau}{\longrightarrow} \cdots\right)
$$

Then Prest et al. [12] show that $S$ is always indiscrete. Now, in our case, $R$ has global dimension 1 , and therefore all of the $M_{k} R$ also have global dimension 1 since they are Morita equivalent to $R$. Now, if we take a pair of $m \times m$ matrices $A, B$ over $S$ with $A B=0$, then we can choose $k$ large enough such that $A, B$ are actually matrices over $M_{n^{k}} R$, and $A B=0$ as such matrices. Then Lemma 3.7 shows that there is a matrix $X$ over $M_{n^{k}} R$, and hence over $S$, with $A X=A$ and $X B=0$. Thus Lemma 3.7 implies that $S$ has weak dimension $\leq 1$, and $S$ cannot be von Neumann regular because $R$ is not (see [12, p. 359]).

The indiscrete rings of [12], of which our counterexample $S$ is one, have been generalized by Garkusha and Generalov [5] to the class of almost regular rings, in which all (left or right) finitely presented modules are FP-injective. The indiscrete rings are the simple almost regular rings.

We also note that the ring $S$ of Theorem 3.5 is in fact weakly semihereditary in the sense of Cohn [2, p. 13]. This means that if $A$ and $B$ are (not necessarily square) matrices such that $A B=0$, then there is an idempotent matrix $E$ such that $A E=A$ and $E B=0$. Since hereditary implies weakly semihereditary, each $M_{n}(R)$ in the above proof is weakly semihereditary, and so the same argument shows that $S$ is as well. One can then use (the left module version of) Lemma 3.7 to see that weakly semihereditary implies weak dimension $\leq 1$.

We now turn to some questions we have not been able to answer. First of all, the stable homotopy category in topology is more like $\mathcal{D}(R)$ for a graded ring $R$ (or, better yet, a differential graded algebra $R$ ), though, it must be stressed, these categories are still much simpler than the stable homotopy category. We have not considered the generating hypothesis for these $R$.

We could ask whether there is a ring $R$ that satisfies the generating hypothesis for right $R$-modules but not left $R$-modules. Such a ring could not be von Neumann regular, of course.

Also, recall that there is a conditionally convergent spectral sequence whose $E_{2}$ term is $\operatorname{Ext}_{R}^{* *}\left(H_{*} P, H_{*} Q\right)$ converging to $\mathcal{D}(R)(P, Q)_{*}$. It seems intuitively evident that for the strong generating hypothesis to hold, this spectral sequence must collapse to the 0 -line for perfect complexes $P$ and $Q$. This is in fact true, since in this case $R$ is von Neumann regular, hence coherent, so the homology groups $H_{*} P$ are finitely presented modules and therefore projective.

However, the situation for $R$ satisfying the generating hypothesis but not the strong generating hypothesis is less clear. To satisfy the generating hypothesis, it must be that every element of $\operatorname{Ext}^{S, *}\left(H_{*} P, H_{*} Q\right)$ with $s>0$ does not survive the spectral sequence. But in order not to satisfy the strong generating hypothesis, there must be an element of $\operatorname{Hom}^{*}\left(H_{*} P, H_{*} Q\right)$ for some perfect $P$ and $Q$ that supports a differential. It would be intriguing to understand how this happens.

Finally, one could define $R$ to satisfy the $n$-fold generating hypothesis if whenever $f_{1}, \ldots, f_{n}$ are composable maps of perfect complexes such that $H_{*}\left(f_{i}\right)=0$ for all $i$, 
then $f_{n} \circ \cdots \circ f_{1}=0$ in $\mathcal{D}(R)$. If we ask for this condition to hold for all $n$-tuples of composable maps with $H_{*} f_{i}=0$, not just maps between perfect complexes, then the second author has shown in his thesis, using work of Christensen [1], that $R$ has projective dimension $\leq n$. One could then ask for an analogous characterization of rings $R$, probably in terms of weak dimension, that satisfy the $n$-fold generating hypothesis, or some strong version of the $n$-fold generating hypothesis.

\section{References}

1. Daniel Christensen, J.: Ideals in triangulated categories: phantoms, ghosts and skeleta. Adv. Math. 136(2), 284-339 (1998) MR MR1626856 (99g:18007)

2. Cohn, P.M.: Free rings and their relations. London Mathematical Society Monographs, vol. 19, 2nd edn. Academic (Harcourt Brace Jovanovich Publishers), London, (1985) MR MR800091 (87e:16006)

3. Faith, C.: Rings and things and a fine array of twentieth century associative algebra. In: Mathematical Surveys and Monographs, vol. 65, American Mathematical Society, Providence (1999) MR MR1657671 (99j:01015)

4. Freyd, P.: Stable homotopy. In: Proceedings of Conference Categorical Algebra (La Jolla, Calif., 1965), pp. 121-172 Springer, New York, (1966) MR MR0211399 (35\#2280)

5. Garkusha, G.A., Generalov, A.I.: Duality for categories of finitely presented modules. Algebra i Analiz 11(6), 139-152 (1999) Translation in St. Petersburg Math. J. 11(6) (2000), 1051-1061 MR MR1746072 (2001a:16005)

6. Goodearl, K.R.: von Neumann regular rings, 2 edn. R. E. Krieger Malabar, (1991) MR MR1150975 (93m:16006)

7. Hovey, M.: On Freyd's generating hypothesis. Q. J. Math. (to appear) doi:10.1093/qmath/hal013

8. Lam, T.Y.: Lectures on modules and rings. In: Graduate Texts in Mathematics, vol. 189, Springer, New York, (1999) MR MR1653294 (99i:16001)

9. Lockridge, K.: The generating hypothesis in the derived category of $R$-modules. J. Pure Appl. Algebra 208(2), 485-495 (2007) doi:10.1016/j.jpaa.2006.01.018

10. Nicholson, W.K., Yousif, M.F.: Principally injective rings. J. Algebra 174(1), 77-93 (1995) MR MR1332860 (96i:16005)

11. Puninski, G., Rothmaler, P.: When every finitely generated flat module is projective. J. Algebra 277(2), 542-558 (2004) MR MR2067618 (2005c:16004)

12. Prest, M., Rothmaler, P., Ziegler, M.: Absolutely pure and flat modules and "indiscrete" rings. J. Algebra 174(2), 349-372 (1995) MR MR1334216 (96d:16002)

13. Sandomierski, F.L.: Nonsingular rings. Proceedings of American Mathematical Society 19, 225-230 (1968) MR MR0219568 (36 \#2648)

14. Stenström, B.: Coherent rings and FP-injective modules. J. London Math. Soc. 2(2), 323-329 (1970) MR MR0258888 (41 \#3533) 